\title{
Airway Management of Retrosternal Goiters in 22 Cases in a Tertiary Referral Center
}

This article was published in the following Dove Press journal:

Therapeutics and Clinical Risk Management

\author{
Yuanming Pan' \\ Chaoqin Chen' \\ Lingya $\mathrm{Yu}^{2}$ \\ Shengmei Zhu' \\ Yueying Zheng' \\ 'Department of Anesthesiology, The \\ First Affiliated Hospital, School of \\ Medicine, Zhejiang University, Hangzhou \\ 3I0003, People's Republic of China; \\ ${ }^{2}$ Department of Radiology, The First \\ Affiliated Hospital, School of Medicine, \\ Zhejiang University, Hangzhou 310003, \\ People's Republic of China
}

Background: The present study aimed to investigate the incidence and extent of difficult airway management in patients with massive retrosternal goiter.

Design: An 8-year retrospective analysis was performed to identify patients who underwent massive retrosternal thyroidectomy. A total of 22 cases were identified as giant retrosternal goiter, followed by a review of each patient's preoperative computerized tomography imaging.

Interventions: There were no cases of failed intubation. Twenty patients underwent uneventful tracheal intubation using direct laryngoscopy or Glidescope. Thirteen patients received a muscle relaxant intravenously, and two patients were induced with sevoflurane. Five patients underwent awake tracheal intubation, including awake fiberoptic intubation in three patients. Before entering the operating theatre, the remaining two patients underwent oral tracheal intubation with Glidescope in the emergency department.

Results: Two patients had tracheal intubation before they entered the operating theatre. Once entering vocal cords, tracheal intubation can pass beyond the site of the tracheal obstruction without difficulty. One patient died because of serious perioperative bleeding owing to the adhesion between the retrosternal goiter and large vessel within the thoracic cavity. One patient experienced dyspnea after extubation and was intubated again.

Conclusion: Intravenous induction of muscle relaxant using laryngoscopy or Glidescope is feasible in patients with massive benign retrosternal goiter. The incidence of difficult intubation and postoperative tracheomalacia is likely too rare. Furthermore, perioperative bleeding and postoperative airway complication seem frequent.

Keywords: airway management, anesthesia, retrosternal goiter, postoperative tracheomalacia

\section{Introduction}

We present our clinical experience on the management of retrosternal goiters.

The retrosternal goiter was defined in numerous ways. The most widely accepted definition was that more than $50 \%$ of the enlarged thyroid gland is below the suprasternal notch. ${ }^{1}$ It was well documented that the incidence of retrosternal goiters were $0.02 \%-15 \% .^{2-4}$ Most retrosternal goiters are secondary, which combine with cervical thyroid and continue growth downward along the plane of the cervical and mediastinal fascia. Based on the different location in three longitude compartments of the mediastinum, the retrosternal goiters are classified into two classes: anterior substernal goiter and posterior substernal goiter, which is mainly coupled with the different surgical approach. ${ }^{5}$ In mediastinum as a confined space, the enlarged substernal goiters compressed onto tracheal, esophagus, and even vital large vessels and
Correspondence: Shengmei Zhu; Yueying Zheng

Department of Anesthesiology, The First

Affiliated Hospital, School of Medicine,

Zhejiang University, Hangzhou 31003,

People's Republic of China

Tel +86- I 3777408863

Email 1507I28@zju.edu.cn
Therapeutics and Clinical Risk Management 2020:16 1267-1273

DovePress in $\square$ 
appeared high incidence of malignancy. So substernal goiters needed to be removed. As referenced, a standard cervical incision was feasible for the majority of retrosternal goiter, but a sternal split or thoracotomy or combined cervical with right posterolateral thoracotomy would be needed in some special patients. ${ }^{5}$

There were few studies investigating the role of enlarged thyroid in complicating airway management. ${ }^{6-8}$ Although the results disagreed on the risk factors for difficult airway among goiter patients, the consensus was that the classical assessment criteria (mouth opening, the Mallapati score, short neck, neck mobility, thyromental distance, and retrognathic mandible) were still reliable in predicting the difficult airway. ${ }^{6,7}$ Retrosternal goiter is not a common cause of different degrees of tracheal compression and tracheomalacia. All of these may lead to difficult intubation or postoperative tracheal collapse. ${ }^{7}$ Thus, we performed a retrospective review of the anesthetic management of patients with different extents of retrosternal goiter and examined the diversity of practice at our institution to determine whether airway management was unnecessarily concerned.

\section{Methods}

As this was a retrospective audit, formal ethical approval was not sought. However, the project was agreed upon by the institution. All thyroidectomies performed from January 2009 to January 2016 were identified using the Operating Room Management Information System (Medical System). This article adheres to the Strengthening the Reporting of Observational Studies in Epidemiology (STROBE) guidelines. ${ }^{9}$ Then we examined electronic anesthetic charts and clinical records and selected patients with a diagnosis of massive goiter or retrosternal goiter. These cases were further cross-referenced with the hospital picture archiving to identify retrosternal extension by using computerized tomography (CT) images via a single radiologist.

Data collected were included the patient characteristics (age, sex, height, and weight) and American Society of Anesthesiologists' (ASA) Physical Status scores. We focused on the information related to anticipated difficult airway, including presenting symptoms (dyspnea, dysphagia, stridor, voice change, venous congestion located in the superior vena cava), anesthetic airway assessment, and especially the planned anesthetic management and subsequent induction of anesthesia and intubation. In addition, perioperative complications were recorded.

Blood testing was performed for thyroid function and autoantibodies. Most cases had an indirect laryngoscopy to ascertain vocal cord function preoperatively. CT scan findings were recorded as part of this study and included thyroid size in the longitudinal axis and horizontal axis, the extent of thyroid gland descent into the thoracic cavity, and the diameter of the narrowest trachea, as well as tracheal deviation from the midline. In the anesthetic documentation, we collected the technique of the induction of general anesthesia (intravenous or inhalation), tracheal intubation (awake or post-induction), Cormack and Lehane grade of laryngoscopy, difficulty or failed intubation, and tracheal extubation.

Operative data such as procedure performed (total or hemithyroidectomy), need for sternotomy (total or partial), the volume of intraoperative blood loss, the need for blood transfusion, and the occurrence of evident tracheomalacia were collected. Postoperative data included the need for reoperation, postoperative care admission (planned or unplanned), and identification of recurrent laryngeal nerve (RLN) palsy was collected.

\section{Results \\ General Data}

14,652 patients underwent thyroidectomy from January 2009 to January 2016 at the First Affiliated Hospital, School of Medicine, Zhejiang University, Hangzhou, China. Among these patients, 34 patients were documented as having an RSG. A total of 22 cases were identified. The enlarged retrosternal thyroid gland was descended to the aortic arch or beyond. Among the 22 patients, 7 were males, and 15 were females with a median age of 62 years (range, 50-77 years), and the history of illness was ranged from 24 to 360 months. The main clinical features of the patients are shown in Table 1.

\section{Thyroid Function Tests}

Thyroid function was normal in four cases; however, thyroid peroxidase antibodies were elevated in eight patients, the thyroid-stimulating hormone was elevated in one case and decreased in six cases, free triiodothyronine and free thyroxin were elevated in two cases, and free thyroxin was decreased in two patients. 
Table I General Clinical Characteristics of Patients $(n=22)$

\begin{tabular}{|l|l|}
\hline Age (Years) & $\mathbf{6 2}$ (50-78) \\
\hline $\begin{array}{l}\text { Sex (number of patients) } \\
\text { Male }\end{array}$ & 7 \\
Female & 15 \\
\hline $\begin{array}{l}\text { Tracheal deviation from midline (mm, number } \\
\text { of patients)) } \\
\text { I0-20 }\end{array}$ & \\
$20-30$ & 14 \\
$>30$ & 4 \\
\hline $\begin{array}{l}\text { Minimum tracheal diameter (mm) } \\
\text { Clinical symptoms (number of patients) }\end{array}$ & 4 \\
\hline $\begin{array}{l}\text { Asymptomatic } \\
\text { Dysphagia }\end{array}$ & $6.4(2-16.4)$ \\
\hline $\begin{array}{l}\text { Dyspnea } \\
\text { Dysphonia }\end{array}$ & 12 \\
\hline $\begin{array}{l}\text { Degree of mediastinal extension (number of } \\
\text { patients) }\end{array}$ & 8 \\
$\begin{array}{l}\text { To aortic arch } \\
\text { Between aortic arch and carina of trachea } \\
\text { To carina of trachea }\end{array}$ & 1 \\
\hline
\end{tabular}

\section{B-Scan Ultrasound Examination}

Fourteen cases were presented with bilateral thyroid nodular lesions with a maximum nodule diameter $>10 \mathrm{~cm}$, and eight patients were presented with bilateral diffuse thyroid lesions with a nodule formation.

\section{Neck and Chest CT Examination}

The mean major axis diameter of the thyroid was $114.2 \pm$ $19.5 \mathrm{~mm}$, and the mean minor axis diameter was $63.6 \pm$ $14.4 \mathrm{~mm}$. The length of the tracheal compression was 62.9 $\pm 30.1 \mathrm{~mm}$. The minimal tracheal diameter ranged from 2-16.4 $\mathrm{mm}$, and the degree of compression at the narrowest point was $>60 \%$. The mean tracheal deviation from midline was $18.7 \pm 9 \mathrm{~mm}$, with nine patients having a tracheal deviation of 10-20 mm, four patients having a tracheal deviation of 20-30 mm, and four patients having a tracheal deviation $>30 \mathrm{~mm}$. Among twenty-two patients, substernal goiters in 21 patients located in anterior mediastinal except for one patient with anterior crossed-over substernal goiters from right to left.

\section{Bronchoscopy}

Three patients underwent bronchoscopy before surgery. External pressure stenosis in the upper right trachea was found in one patient, and total airway stenosis was found in one patient.

\section{Preanesthetic Assessment}

Twenty patients were classified as ASA II, and two patients were classified as ASA III. Twenty-two patients had an extent of mouth opening of $>3 \mathrm{~cm}$ and a thyromental distance of $>6 \mathrm{~cm}$ with Mallampati classes I or II.

\section{Anesthesia}

Thirteen patients were anesthetized by intravenous injection of propofol. Two patients were anesthetized by sevoflurane inhalation. Five patients were given awake tracheal intubation in which two patients completed endotracheal intubation under laryngoscopic guidance and three patients completed intranasal tracheal intubation with fiberoptic bronchoscope guidance. During the intravenous induction of anesthesia, after confirming no difficulty with mask ventilation, a muscle relaxant (rocuronium or cisatracurium besylate) was injected intravenously to assist intubation. Standard laryngoscopes or video laryngoscopes were used for laryngoscopy. One case anesthetized by sevoflurane inhalation was completed by laryngoscopy under tracheal intubation. Another case with sevoflurane anesthesia was completed under nasal endotracheal intubation under the guidance of a fiberoptic bronchoscope. For all patients undergoing awake tracheal intubation, complete fiberoptic bronchoscopy-guided intranasal intubation or laryngoscopy-guided tracheal intubation was completed after cricothyrotomy with topical anesthesia $(2-3 \mathrm{~mL}$ of $2 \%$ lidocaine) supplemented with fentanyl or dexmedetomidine. Muscle relaxants were supplemented immediately after successful intubation. Intraoperative anesthesia was maintained using propofol, remifentanil, or sevoflurane. Among the twenty-two patients, two patients were brought into the operating room with tracheal tubes. One patient was given emergency bedside tracheal intubation in the ward for sudden dyspnea before surgery and one patient was given tracheal intubation after emergency admission due to syncope. Among the eight patients who had preoperative symptoms of dyspnea, intravenous anesthesia was used in the five patients, awake tracheal intubation was used in two patients, and one patient was brought into the operating room with a tracheal tube.

A steel wire ring-reinforced tracheal tube with an inner diameter of $6.0-8.0 \mathrm{~mm}$ was used. In patients with a tracheal deviation from the midline of $>30 \mathrm{~mm}$, 
Table 2 Anesthesia Management of Patients

\begin{tabular}{|l|l|}
\hline Anesthesiology Parameter & $\mathbf{n}$ (Patients) \\
\hline $\begin{array}{l}\text { Method of anesthesia induction } \\
\text { Intravenous induction }\end{array}$ & 13 \\
Inhalation induction & 2 \\
\hline Awake tracheal intubation & 5 \\
Laryngoscopy or video laryngoscopy & 2 \\
Fiber optic bronchoscopy & 3 \\
Tracheal tube brought into operation room & 2 \\
\hline
\end{tabular}

a tracheal tube with an inner diameter of 6.0-7.0 mm was selected. In patients with the narrowest tracheas of 2-4 $\mathrm{mm}$, a tracheal tube with an inner diameter of 6.5-7.5 mm was selected. After the tracheal tube was inserted into the glottis, it passed the tracheal stenosis uneventfully, and no patient had difficulty with mechanical ventilation. The anesthesia management of the patients is summarized in Table 2.

\section{Intraoperative and Postoperative Conditions}

Thirteen patients underwent retrosternal goiter resection by cervical approach, and eight patients required combined with partial sternal split to complete the operation, and only one patient changed from partial sternal split to total sternal split temporarily. Two patients received intraoperative red blood cell transfusions. One patient had severe bleeding due to extreme adhesion of the thyroid gland to the surrounding blood vessels and difficulty in separation, and emergency intraoperative extracorporeal circulation was used. Because of the large wound surface and severe leakage of the blood, there was more bleeding. It was challenging to maintain stable postoperative circulation, making it hard to discharge the patient. Ten patients were planned to be transferred to the intensive care unit for postoperative tracheal catheter observation. After the cuff leak test evaluation, oxygen saturation was maintained above $95 \%$, and the patients were extubated. One patient experienced dyspnea after extubation and was intubated again. No patient required another tracheal intubation due to postoperative hemorrhage and no patient developed symptoms of recurrent laryngeal nerve paralysis.

In terms of postoperative pathology, there were fifteen cases of nodular goiter, five cases of adenomatoid nodules, and two cases of follicular adenoma. The intraoperative and postoperative conditions of the patients are summarized in Table 3.
Table 3 Intraoperative and Postoperative Condition of Patients

\begin{tabular}{|l|l|}
\hline Intraoperative and Postoperative Condition & $\mathbf{n}$ (Patients) \\
\hline Intraoperative sternotomy & 9 \\
\hline Intraoperative blood transfusion & 2 \\
\hline Pathological findings & \\
Nodular goiter & 15 \\
Adenomatoid nodules & 5 \\
Follicular adenoma & 2 \\
\hline Postoperative complications & \\
Recurrent laryngeal nerve paralysis & 0 \\
Bleeding & 0 \\
Tracheomalacia & 0 \\
Dyspnea & 1 \\
\hline
\end{tabular}

\section{Discussion}

Massive retrosternal goiter not only increases the difficulty of surgery, postoperative complications, and mortality but also poses a challenge to perioperative anesthesia management. Massive retrosternal goiter causes tracheal compression and deviation, which may cause a difficult airway and put stress on the cardiovascular system, leading to circulatory failure or postoperative tracheomalacia. Currently, the literature on perioperative anesthesia management for patients with huge retrosternal goiter is mostly limited to case reports. ${ }^{10,11}$

For patients with massive retrosternal goiter, the anesthesiologist should first focus on whether there is a difficult airway and then select an anesthesia induction protocol and tracheal intubation method. Most researchers believe that patients with massive retrosternal goiter often have difficult airways, but recent research results do not support this view. In a study of anesthesia induction protocols in 62 cases of goiter surgery in patients with tracheal compression by Findlay et al, the standard intravenous anesthesia induction protocol was used to complete tracheal intubation in $85 \%$ of patients. $^{12}$ Bennett et al showed that only 6 out of 1,969 patients who underwent through thyroid surgery had difficult airways for tracheal intubation. ${ }^{13}$ Of patients expected to have difficult airways before anesthesia, 87\% completed intubation under direct laryngoscopy. ${ }^{14}$ In the present study, four patients with difficult airways on a preoperative evaluation completed tracheal intubation successfully under direct laryngoscopy or video laryngoscopy with intravenous induction of anesthesia. Eighteen patients with a tracheal compression diameter of $<1 \mathrm{~cm}$ also did not have difficult airways. Two patients were 
brought into the operating room with tracheal tubes, in which one patient had a narrowest tracheal inner diameter of about $2 \mathrm{~mm}$, and completed intubation with video laryngoscopy in the emergency department.

According to the literature, conventional intravenous anesthesia induction, inhalation anesthesia induction, and awake fiberoptic bronchoscopy-guided tracheal intubation (oral or intranasal) are the most commonly used methods in patients with retrosternal goiter. ${ }^{15}$ The intravenous anesthesia induction protocol is not contraindicated in patients with retrosternal goiter, nor is it the cause of the difficulty in direct laryngoscopic exposure. Of the 22 patients in the present study, 13 patients underwent tracheal intubation using intravenous anesthesia induction. Laryngoscopic exposure can be facilitated by the application of muscle relaxants during the intravenous anesthesia induction protocol. In specific patients, especially those who cannot tolerate hypoxia or be awake, awake fiberoptic bronchoscope-assisted endotracheal intubation protocols are not tolerated and an intravenous anesthesia induction protocol is necessary. ${ }^{14}$ Switching to intravenous anesthesia induction is often successful after the failure of planned inhalation anesthesia induction or awake fiberoptic bronchoscopic intubation. ${ }^{16}$ The role of awake fiberoptic bronchoscope-guided tracheal intubation under challenging airways is generally recognized by anesthesiologists. However, for patients with symptoms such as wheezing or airway obstruction, the fiberoptic bronchoscope placement causes airway blockage that to result in the patient being unable to tolerate hypoxia. Therefore, the application of this technique in patients with severe tracheal obstruction is controversial and even considered contraindicated. ${ }^{17}$ In addition, skillful application of this technique requires long training and has a relatively steep learning curve. In anesthesia induction protocols for patients with airway obstruction, inhalation induction may be a more accepted method. However, inhalation anesthesia takes time, and the timing of laryngoscope insertion needs to be controlled appropriately, as inserting too early can cause airway complications, and insert too late can cause hypoxemia. Therefore, inhalation anesthesia induction involves a specialized skill, and thus it requires professional training. In a study by Dempsey et al, only 1 out of 34 patients with massive retrosternal goiter underwent through inhalation anesthesia had difficulty with glottic exposure and required an emergency tracheotomy. The Cormack-Lehane classification in the latter part of the operation with direct laryngoscopy was grade 2. ${ }^{15}$ Taken together with our study, it concluded that intravenous anesthesia induction and traditional laryngoscopy or video laryngoscopy could be used more safely in patients with benign massive retrosternal goiter. However, due to the limited number of patients in this cohort, a study with larger sample size is still needed for further clinical validation.

Patients with massive retrosternal goiter are often accompanied by severe tracheal compression and deviation. In this study, 18 patients had a diameter of tracheal compression of $<1 \mathrm{~cm}$, so the selection of tracheal tube size is another issue considered by anesthesiologists. Based on the results of the present study, benign retrosternal thyroid tumors do not affect the selection of tracheal tube size, and the patient's size can still be the principal basis for the selection of tracheal tube size. The steel-wire tracheal tube (inner diameter 7.0-7.5 mm) selected in the present study was successfully inserted into the glottis and passed through the stenosis. Unlike the invasive growth of malignant tumors, benign thyroid tumors have only external compressive forces that narrow the trachea. Because of its flexibility and mobility, the steel-wire tracheal tube can still open and pass the site of tracheal stenosis without affecting positive pressure ventilation even if the trachea is severely compressed. However, malignant thyroid tumors may infiltrate the trachea, and surgery may require a tracheotomy.

Besides, although massive retrosternal goiters are located in the mediastinum, they originate in the neck and, therefore, represent different anesthesia risks than mediastinal tumors. Benign retrosternal goiter rarely carries the risk of serious cardiovascular events due to tumor collapse resulting in airway obstruction or compression of the cardiovascular system after anesthesia induction. Preoperative visualization of the blood supply of the tumor on chest CT can help to distinguish between retrosternal goiter and mediastinal tumors. Therefore, preoperative CT is also vital in patients with retrosternal goiter. Before an operation, the anesthesiologist also needs to read the CT carefully and entirely communicate with the surgeon to know whether the tumor is benign or malignant and get other necessary information.

Tracheomalacia is a major concern after massive retrosternal goiter resection. After resection, the trachea may collapse due to loss of support from the surrounding tissues that cause suffocation. This view is currently being questioned. An analysis of 34 papers and 2,426 patients with retrosternal goiter by Huins et al showed that the incidence of tracheomalacia was $<1 \%$. ${ }^{8}$ Chauhan et al and Sudan et al reported that the incidence of tracheomalacia was about $0.05 \% .^{18,19}$ A postoperative investigation by Findlay et al on patients with goiter with severe tracheal compression found that the incidence of postoperative tracheomalacia is zero. ${ }^{12}$ However, the incidence of tracheomalacia after resection of massive retrosternal goiter extending to the aortic arch can reach $10 \%{ }^{8}$ In the present 
study, ten patients were planned to be sent to the ICU after the operation, one of which needed intubation again after the extubation of the tracheal tube due to dyspnea. The indications for extubation should be strictly controlled after the operation, and the tracheal tube should be removed after the patient is fully awake.

The limitations of the present study are as follows: (1) This is a retrospective study with a small number of cases. It is limited by the incidence of massive retrosternal goiter, and no better solution to this problem exists. (2) The pathological results of all the patients in this study indicated benign goiters; therefore, the scope of the conclusions of this paper is limited. The incidence of difficult airway in benign massive retrosternal goiter is low, and tracheal tubes can pass more easily through a narrowed trachea. Nevertheless, we still encourage anesthesiologists and surgeons to fully communicate before surgery to obtain a clear understanding of airway management and to be more fully prepared for airway emergencies.

\section{Conclusion}

The results of the present study show that adequate communication between the anesthesiologist and the surgeon may prevent difficult airways in patients with benign massive retrosternal goiter. They can choose between intravenous anesthesia induction protocols and laryngoscopy for tracheal intubation, and they should exercise vigilance for tracheomalacia.

\section{Ethics Approval and Informed Consent}

Ethics approval was obtained from the Clinical Research Ethics Committee of the First Affiliated Hospital, College of Medicine, Zhejiang University (reference numbers:2016141-1). Informed consent could not be obtained. The authors declare that the work described has been carried out in accordance with the Declaration of Helsinki of the World Medical Association revised in 2013 for experiments involving humans. This study was retrospective, and the need for informed consent was waived. Adequate measures to ensure data protection, confidentiality, patients' privacy and anonymization were taken into account in compliance with China National Committee for Data Protection laws.

\section{Acknowledgments}

We thank Dr. Fuqiang Li and Dr. Feng Zhu (the First Affiliated Hospital, College of Medicine, Zhejiang University) for revising the manuscript.

\section{Author Contributions}

All authors made a significant contribution to the work reported, whether that is in the conception, study design, execution, acquisition of data, analysis and interpretation, or in all these areas; took part in drafting, revising or critically reviewing the article; gave final approval of the version to be published; have agreed on the journal to which the article has been submitted; and agree to be accountable for all aspects of the work.

\section{Funding}

This work was supported by the Project of Experimental Animal Science and Technology Plan of Zhejiang Province [grant numbers2018C37116, Yueying Zheng].

\section{Disclosure}

The authors report no conflicts of interest in this work.

\section{References}

1. Glazer GM, Axel L, Moss AA. CT diagnosis of mediastinal thyroid. AJR Am J Roentgenol. 1982;138(3):495-498. doi:10.2214/ ajr.138.3.495

2. Katlic MR, Grillo HC, Wang C-A. Substernal goiter: analysis of 80 patients from Massachusetts General Hospital. Am J Surg. 1985;149 (2):283-287. doi:10.1016/S0002-9610(85)80086-6

3 . Reeve TS, Rundle FF, Hales IB, et al. The investigation and management of intrathoracic goiter. Surg Gynecol Obstet. 1962;115:222-229.

4. Sanders LE, Rossi RL, Shahian DM, Williamson WA. Mediastinal goiters: the need for an aggressive approach. Arch Surg. 1992;127 (5):609-613. doi:10.1001/archsurg.1992.01420050137018

5. Hubbard J, Inabnet WB, Lo C-Y. Endocrine Surgery: Principles and Practice. Springer Science \& Business Media; 2009.

6. Chaoyang L, Yuer S. Surgical Treatment of Intrathoracic Goiter. Chin J Thorac Cardiovasc Surg. 1996;2.

7. Testini M, Nacchiero M, Miniello S, et al. Management of retrosternal goiters: experience of a surgical unit. Int Surg. 2005;90 (2):61-65.

8. Huins CT, Georgalas C, Mehrzad H, Tolley NS. A new classification system for retrosternal goitre based on a systematic review of its complications and management. Int $J$ Surg. 2008;6(1):71-76. doi:10.1016/j.ijsu.2007.02.003

9. von Elm E, Altman DG, Egger M, Pocock SJ, Gøtzsche PC, Vandenbroucke JP. Strengthening the Reporting of Observational Studies in Epidemiology (STROBE) statement: guidelines for reporting observational studies. BMJ (Clinical Research Ed). 2007;335 (7624):806-808. doi:10.1136/bmj.39335.541782.AD

10. Patil S, Bhayani S. Airway obstruction caused by the systemic inflammatory syndrome associated with trauma and retrosternal goitre. Resuscitation. 2009;80(10):1095-1096. doi:10.1016/j. resuscitation.2009.06.018

11. Dave ST, Kamath SK, Shetty AN, Naik LD. Anaesthesia management for subtotal thyroidectomy in a case of multinodular goitre with retrosternal extension and superior vena caval syndrome. J Postgrad Med. 2001;47(3):219.

12. Findlay JM, Sadler GP, Bridge H, Mihai R. Post-thyroidectomy tracheomalacia: minimal risk despite significant tracheal compression. Br J Anaesth. 2011;106(6):903-906. doi:10.1093/bja/ aer062 
13. Bennett AM, Hashmi SM, Premachandra DJ, Wright MM. The myth of tracheomalacia and difficult intubation in cases of retrosternal goitre. J Laryngol Otol. 2004;118(10):778-780. doi:10.1258/ 0022215042450751

14. Gilfillan N, Ball CM, Myles PS, Serpell J, Johnson WR, Paul E. A cohort and database study of airway management in patients undergoing thyroidectomy for retrosternal goitre. Anaesth Intensive Care. 2014;42(6):700-708. doi:10.1177/0310057X1404200604

15. Dempsey GA, Snell JA, Coathup R, Jones TM. Anaesthesia for massive retrosternal thyroidectomy in a tertiary referral centre. $\mathrm{Br}$ J Anaesth. 2013;111(4):594-599. doi:10.1093/bja/aet151

16. Loftus PA, Ow TJ, Siegel B, Tassler AB, Smith RV, Schiff BA. Risk factors for perioperative airway difficulty and evaluation of intubation approaches among patients with benign goiter. Ann Otol Rhinol Laryngol. 2014;123(4):279-285. doi:10.1177/0003489414524171
17. Cook TM, Woodall N, Frerk C. Major complications of airway management in the UK: results of the Fourth National Audit Project of the Royal College of Anaesthetists and the Difficult Airway Society. Part 1: anaesthesia. Br J Anaesth. 2011;106 (5):617-631. doi:10.1093/bja/aer058

18. Chauhan A, Serpell JW. Thyroidectomy is safe and effective for retrosternal goitre. ANZ J Surg. 2006;76(4):238-242. doi:10.1111/ j.1445-2197.2006.03699.x

19. Rahim AA, Ahmed ME, Hassan MA. Respiratory complications after thyroidectomy and the need for tracheostomy in patients with a large goitre. $\mathrm{Br} J$ Surg. 1999;86(1):88-90. doi:10.1046/j.13652168.1999.00978.x

\section{Publish your work in this journal}

Therapeutics and Clinical Risk Management is an international, peerreviewed journal of clinical therapeutics and risk management, focusing on concise rapid reporting of clinical studies in all therapeutic areas, outcomes, safety, and programs for the effective, safe, and sustained use of medicines. This journal is indexed on PubMed Central, CAS,
EMBase, Scopus and the Elsevier Bibliographic databases. The manuscript management system is completely online and includes a very quick and fair peer-review system, which is all easy to use. Visit http://www.dovepress.com/testimonials.php to read real quotes from published authors. 